DOI: $10.34185 / 1991-7848 . i t m m .2020 .01 .013$

\title{
РАЗВИТИЕ ТЕОРИИ И ПРАКТИКИ НЕПРЕРЫВНОЙ ПРОКАТКИ ГОРЯЧЕЙ ПОЛОСЫ
}

Веренев В.В., д.т.н., ст.н.с., Подобедов Н.И., к.т.н., ст.н.с., Мацко С.В., Институт черной металлургии им. З.И. Некрасова НАН Украины, Украина ПАО «Запорожсталь», Украина

Аннотация. Приведены результаты теоретических и опытнопромышленных исследований на стане 1680 в период внедрения непрерывной прокатки в трех парах черновых клетей. С помощью разработанной математической модели, составленной в абсолютных значениях переменных, раскрыт характер формирования межклетевых натяжений при заполнении толстой полосой каждой из пар черновых клетей и раскатом непрерывной шестиклетевой группы. Учет упругих крутильных колебаний линий привода позволил прояснить роль высокочастотной составляющей в переходных процессах и повысить точность определения динамических нагрузок на 15-20 \%. Раскрыт процесс транспортного переноса (запаздывания) толщины полосы от клети к клети в взаимосвязи с межклетевыми натяжениями. Показана количественная связь формирующихся натяжений по мере заполнения непрерывной группы полосой с образованием утолщенных концевых участков готового проката. Установлены такие сочетания возмущений (по толщине и температуре раската), которые приводят к существенному увеличению межклетевых натяжений и разнотолщинности или их уменьшению. Путем опытнопромышленных измерений установлены особенности взаимодействия черновых клетей через толстую полосу.

КлючевЫе слова: НЕПРЕРЫВНАЯ ПРОКАТКА, ЗАХВАТ ПОЛОСЫ, УДАРНЫЕ НАГРУЗКИ, ПЕРЕХОДНЫЙ ПРОЦЕСС, МЕЖКЛЕТЕВЫЕ НАТЯЖЕНИЯ, РАЗНОТОЛЩИННОСТЬ.

Введение. На станах «Слябинг-1680» металлургического комбината «Запорожсталь» на рубеже веков внедрялись новые технологии прокатки. В частности, в черновых клетях, не предусмотренных для этой цели, пары клетей ДУО - № 1, № 2 - 3 и № 3 - 4 перевели на непрерывную прокатку транзитом слябов удвоенной массы и длины - по технологии «слиток - сляб - рулон». При 
Ministry of Education and Science of Ukraine

The National Metallurgical Academy of Ukraine, Dnipro, 17 - 19 March, 2020

этом клети № 1 и 2 содержали нерегулируемые двигатели переменного тока, клети ДУО, № 3 и 4 - двигатели постоянного тока. Планировалось внедрение перед чистовой группой клетей промежуточного перемоточного устройства типа «Койлбокс». Отмеченное выше вызвало потребность в решении новых задач с учетом обновленной специфики производства. Например, определение динамических усилий в полосе и оборудовании при захвате и непрерывной прокатке толстой полосы в каждой из трех пар клетей; динамики заполнения и освобождения полосой непрерывной шестиклетевой группы в взаимосвязи с продольной разнотолщинностью проката и др.

В результате совместных опытно-промышленных и теоретических исследований ИЧМ совместно с комбинатом «Запорожсталь» получены новые решения, закономерности и соотношения, которые подтвердили курс руководства комбината на внедрение новых технологий.

Основной материал. Необходимость решения поставленных задач обусловила развитие новых подходов к теоретическим исследованиям динамики непрерывной прокатки горячей полосы. Они состоят в том, что динамика процесса прокатки и работы оборудования в переходных режимах рассматривается в единстве и взаимосвязи технологических параметров и колебательных явлений в упругой системе клети и электромеханической линии главного привода валков, в очаге деформации и в межклетевых промежутках и в конечном итоге в продольной разнотолщинности прокатываемой полосы [1].

Конкретно это состояло в следующем.

1. Математическая модель динамики технологического взаимодействия через полосу при непрерывной прокатке изложена в абсолютных значениях переменных в отличие от традиционно применяемых моделей в отклонениях.

2. Учтены упругие колебания клетей и крутильные колебания линий главного привода. Это позволило раскрыть высокочастотную составляющую колебаний технологических параметров, которые на станах горячей прокатки не измеряются: межклетевые натяжения, опережение, скорость выхода и входа полосы и др. Кроме того, на 15-20 \% повысить точность определения максимальных значений межклетевых усилий и отклонения толщины полосы 
International scientific and technical conference Information Technologies in Metallurgy and Machine building - ITMM 2020

по сравнению с «жесткой» моделью, когда податливость линий привода и крутильные колебания не учитываются.

3. Учтено и программно реализовано уравнение транспортного переноса (запаздывания) толщины полосы. Раскрыта его роль и механизм проявления при заполнении 6-клетевой непрерывной группы полосой и во время прокатки.

4. Впервые раскрыты и установлены закономерности динамики совместного формирования межклетевых натяжений и продольной разнотолщинности прокатываемой полосы по мере ее последовательного захвата и прокатки в 6-клетевой непрерывной группе. Разработанная модель в отличие от известных качественных объяснений позволила установить количественную меру утолщения и длину (15-25 мм) концевых участков с учетом различных условий прокатки в режимах заполнения и освобождения полосой непрерывной группы. С помощью известных моделей в отклонениях данная задача не решается.

5. Математическая модель идентифицирована по данным опытнопромышленных измерений крутящих моментов в линиях привода черновой непрерывной подгруппы клетей № 3-4-5 стана 2000 АО «Северсталь» и в чистовых клетях станов 1680, 1700 и 2500. Результаты решений на модели показывают характер динамики нарастания межклетевого натяжения при захвате полосы в $i$-й клети и за счет этого небольшое (5-15 \%) падение крутящего момента в предыдущей (i-1)-й клети. Результаты решений дают наглядное представление о взаимосвязи динамики формирования межклетевых натяжений и продольной разнотолщинности концевых участков.

6. Получили дальнейшее развитие представления о динамических закономерностях и количественном влиянии технологических возмущений отклонения толщины и температуры подката, скорости прокатки и др., а также параметров оборудования - жесткости клетей и главных линий, на формирование межклетевых натяжений и продольной разнотолщинности готовой полосы. Показано, что имеются сочетания возмущений, когда происходит существенное увеличение межклетевых натяжений и 
Ministry of Education and Science of Ukraine

The National Metallurgical Academy of Ukraine, Dnipro, 17 - 19 March, 2020

разнотолщинности или взаимное парирование их влияния на указанные параметры.

7. Впервые раскрыт характер изменения межклетевых натяжений в чистовой группе при перемещении нажимных винтов с целью регулирования толщины полосы. Вскрытые закономерности позволяют учитывать их при проектировании систем регулирования.

8. Впервые раскрыты закономерности изменения общего коэффициента выравнивания относительной продольной разнотолщинности готовой полосы. Установлено, что общий и частные по клетям коэффициенты выравнивания относительной продольной разнотолщинности в режиме заполнения непрерывной группы клетей полосой существенно отличаются от коэффициентов в режиме установившейся прокатки с заданными натяжениями.

9. Получило развитие познание динамики технологического и динамического взаимодействия пар клетей при непрерывной прокатке толстой полосы (от 150 мм до 70-50 мм). При захвате толстой полосы валками клети 1 в линии привода клети дуо стана 1680 формируется максимальный динамический момент, соизмеримый с моментом при захвате сляба валками клети дуо. Раскрыт механизм образования существенного подпора или натяжения в полосе между этими клетями и определено соотношение скорости валков клетей, когда динамические нагрузки становятся наименьшими.

10. На базе комплекса натурных и теоретических исследований получило развитие новое направление в диагностике состояния технологии и оборудования, основанное на использовании информации о переходных процессах в период захвата полосы валками и взаимодействия клетей через полосу [2].

Выводы. Результаты совместной работы на разных этапах исследований способствовали освоению и внедрению непрерывной прокатки в парах черновых клетей технологии "слиток - сляб - рулон" и устройства «Койлбокс», что повысило эффективность работы широкополосного стана 1680 комбината "Запорожсталь".

Математическая модель и компьютерная программа предназначены для: 
International scientific and technical conference Information Technologies in Metallurgy and Machine building - ITMM 2020

- коррекции существующих и разработки новых деформационноскоростных режимов прокатки в непрерывных группах клетей, а также при освоении новых марок сталей;

- оперативной оценки возможности непрерывной прокатки или предупреждения нежелательных последствий в случаях нештатных ситуаций по технологии (повышенные отклонения параметров) или оборудования (увеличенный износ, временный вывод из работы одной из клетей и др.);

- установления новых закономерностей и взаимосвязей параметров технологии и оборудования;

- оценки выравнивающих свойств продольной разнотолщинности чистовой группой клетей при поступлении повышенных возмущений;

- разработки способов диагностирования состояния технологии и оборудования с учетом его параметров и состояния;

- использования в учебном процессе в ВУЗах при изучении технологии непрерывной прокатки и прокатного оборудования, а также повышения квалификации операторов прокатных станов.

\section{Литература}

1. Веренев В.В., Путноки А.Ю., Подобедов Н.И. Переходные процессы при непрерывной прокатке. Днепр: Літограф. 2017. 116 с.

2. Диагностика и динамика прокатных станов. [В.В, Веренев, В.И. Большаков, А.Ю. Путноки и др.]. Днепропетровск: ИМА-пресс, 2007. - 144 с.

\section{DEVELOPMENT OF THE THEORY AND PRACTICE OF CONTINUOUS ROLLING OF A HOT STRIP}

Verenev Valentyn, Podobedov Mykolai, Matsko Serhii

Annotation. The results of theoretical and pilot studies at the 1680 mill during the introduction of continuous rolling in three pairs of roughing stands are presented. Using the developed mathematical model, compiled in the absolute values of the variables, the nature of the formation of inter-stand tension is revealed when each pair of draft stands is filled with a thick strip and a continuous six-row group is rolled. Taking into account the elastic torsional vibrations of the drive lines made it possible to clarify the role of the high-frequency component in transients 
and to increase the accuracy of determining dynamic loads by $15-20 \%$. The process of transport transfer (delay) of the thickness of the strip from the cage to the cage in relation to intercellular tension is disclosed. A quantitative relationship of the formed tension is shown as the continuous group is filled with a strip with the formation of thickened end sections of the finished product. Such combinations of perturbations (in thickness and temperature of the roll) are established that lead to a significant increase in interstand stresses and thickness differences or their reduction. Through pilot measurements, the features of the interaction of roughing stands through a thick strip are established.

Key words: CONTINUOUS ROLLING, STRIP GRABBING, IMPACT LOADS, TRANSIENT PROCESS, INTERSTAND TENSION, THICKNESS DIFFERENCE.

\section{References}

1. Verenev VV, Putnoki A.Yu., Podobedov N.I. Transients during continuous rolling. Dnipro: Lithograph. $2017.116 \mathrm{~s}$.

2. Diagnostics and dynamics of rolling mills. [V.V, Verenev, V.I. Bolshakov, A.Yu. Putnoki and others.]. Dnepropetrovsk: IMA-press, 2007 .- 144 p. 